\title{
Celiac disease within the villous atrophy syndrome in preschool children
}

\author{
G. Baciu, P. Popa, M. Dobre and A. Nechita \\ Faculty of Medicine and Pharmacy, 'Dunarea de Jos' University of Galati, 35, A.I.Cuza Street, Galati, Romania
}

Villous atrophy syndrome aetiology includes celiac disease, cow milk protein allergy, duodenitis, temporary gluten intolerance, food allergies (to other than cow milk), occasional giardiosis and secondary lactase deficits. This study endeavours to evaluate the incidence of celiac disease in villous atrophy syndrome, to determine optimal age for diagnosis and most frequent signs and symptoms that occur at the onset of the disease.

The study group was made up of 112 children aged between 3 months and 7 years, which were hospitalised over a period of 2 years and exhibited characteristic symptoms, such as prolonged diarrhoea (lasting more than 3 weeks), underweight and/or arrested development, vomiting, lack of appetite, abdominal pains and bloating. All patients were investigated using serum tests for celiac disease and superior digestive endoscopy with duodenum biopsy. The correlation between histopathological aspects (Marsh classification) and clinical patient aspects, i.e. antitransglutaminase and antiendomisium antibody levels, were studied through quantitative correlations between antibody levels and lesion severity.

Clinical manifestations of the malabsorbtion syndrome were caused, in order of frequency, to celiac disease, digestive intolerance to cow milk protein, secondary lactase deficits and postenteritic syndrome. Onset of the disease was in $59.33 \%$ of cases before 1 year of age, and the evolution of the disease was classical in $75 \%$ of cases, most frequently manifesting itself by chronic diarrhoea and underweight. In patients with celiac disease, villous atrophy (Marsh class III a-c) occurs in most underweight and chronic diarrhoea patients $(70 \%$ of cases) or in patients suffering from abdominal pains $(60 \%)$. Over $60 \%$ of patients diagnosed with celiac disease tested positive for at least one of two studied serum markers (antiendomisium and antitransglutaminase antibodies). All celiac disease sufferers who also had Marsh IIIc histological modifications were serum positive for at least one of the investigated serum antibodies. Marsh II histology patients were the fewest to yield serum positive results.

In many studies in children, celiac disease was the most frequent cause of clinical manifestations characteristic of the malabsorbtion syndrome associated with villous atrophy histological modifications.

Of the four diseases characterised by villous atrophy syndrome, celiac disease is most frequently accompanied by paraclinical modifications, which have important repercussions on the organism (anaemia, low iron and magnesium levels and bone growth retardation). 